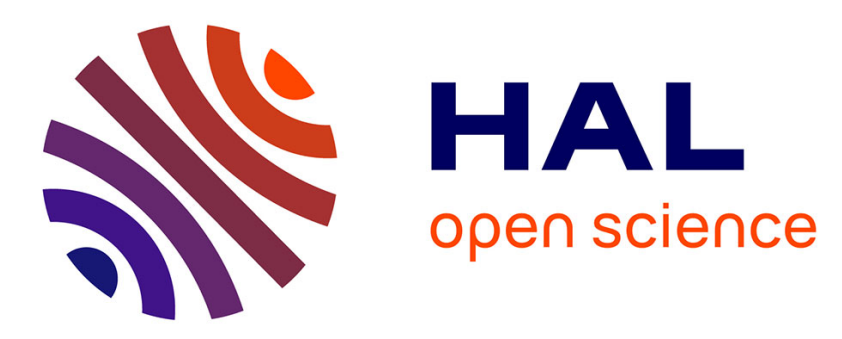

\title{
Finite element analysis of blow-molding and thermoforming using a dynamic explicit procedure
}

Gilles Marckmann, Erwan Verron, Bernard Peseux

\section{To cite this version:}

Gilles Marckmann, Erwan Verron, Bernard Peseux. Finite element analysis of blow-molding and thermoforming using a dynamic explicit procedure. Polymer Engineering and Science, 2001, 41 (3), pp.426-439. 10.1002/pen.10740 . hal-01006898

\section{HAL Id: hal-01006898 \\ https://hal.science/hal-01006898}

Submitted on 24 Oct 2016

HAL is a multi-disciplinary open access archive for the deposit and dissemination of scientific research documents, whether they are published or not. The documents may come from teaching and research institutions in France or abroad, or from public or private research centers.
L'archive ouverte pluridisciplinaire HAL, est destinée au dépôt et à la diffusion de documents scientifiques de niveau recherche, publiés ou non, émanant des établissements d'enseignement et de recherche français ou étrangers, des laboratoires publics ou privés. 


\title{
Finite Element Analysis of Blow Molding and Thermoforming Using a Dynamic Explicit Procedure
}

\author{
G. MARCKMANN, E. VERRON*, and B. PESEUX \\ Laboratoire de Mécanique et Matériaux \\ Division Structures \\ École Centrale de Nantes \\ BP 92101,44321 Nantes cedex 3, France
}

\begin{abstract}
This paper reports on the development of a dynamic finite element procedure for the simulation of blow molding and thermoforming of thermoplastic hollow parts. The Principle of Virtual Work written herein takes inertia effects into account. The heat-softened parison is assumed to be a nonlinear hyperelastic Mooney-Rivlin membrane and is meshed with classical linear triangular finite elements. We adopt the explicit central differences time integration scheme with the special lumping technique. The mold is divided into triangular elements and the contact between the parison and the mold is assumed to be sticky. Therefore, contacted degrees of freedom of the parison are fixed on the solid boundary until the end of the simulation. Performances are highly improved by the use of an adaptive mesh refinement procedure based on a geometric criterion for detection and on the simple addition of a node at the mid-side of the longest edge for subdivision. The method is illustrated through some examples of thermoformed and blow-molded parts. Our results are compared with both experimental and numerical results from literature to validate the present theory.
\end{abstract}

\section{INTRODUCTION}

$\mathrm{T}$ hree different industrial techniques are used to produce thin, hollow thermoplastic parts. These techniques are presented in Fig. 1. The first is the extrusion blow-molding process, which consists of three steps: first the polymer melt is extruded through an annular die to form a parison, then this parison is clamped at its bottom end, and finally it is inflated in the mold (see Fig. la). This process is widely used in the production of irregularly shaped and irregularly sized containers in various industries. The injection stretch blow-molding process of PET bottles also involves three steps: the making of a preform by injection molding, the heating of this preform, and the simultaneous stretching and blowing in the mold (see Fig. Ib). The last process is thermoforming: a previously extruded thermoplastic sheet is clamped, then heated far from its glass transition temperature and formed into a mold cavity using a vacuum pressure (see Fig. 1c). This process is commonly used in the packaging industry. Details on these different techniques can be found elsewhere for blow molding (1) and for thermoforming (2).

*Corresponding author.
In these three processes, a thin plastic membrane is heat-softened and inflated in a cold mold cavity. This paper deals only with the blowing stage of each technique. Thus an analogous approach can be followed in the numerical simulation of these processes. The main goals of industrial process simulation are, first, to predict the final thickness distribution in the molded object or to obtain a desired final thickness distribution in the molded piece, and second, to optimize the processing parameters such as temperature, pressure or mold geometry.

In order to review the papers dealing with the thermoplastic forming simulation, we must first consider works that are focused on the traditional rubber industry and do not explicitly mention blow molding or thermoforming. It is well known that parisons are considered as incompressible hyperelastic or viscoelastic isotropic membranes; therefore, the theoretical work of Green and Adkins (3) on the inflation of rubber membranes is commonly used as the basis of such studies. In the general context of membrane inflation, Hart-Smith and Crisp (4) examined the case of axisymmetric rubberlike membrane inflation using shooting procedures to solve the corresponding two-point boundary value problem. Oden and Sato (5) were the first 
(a)

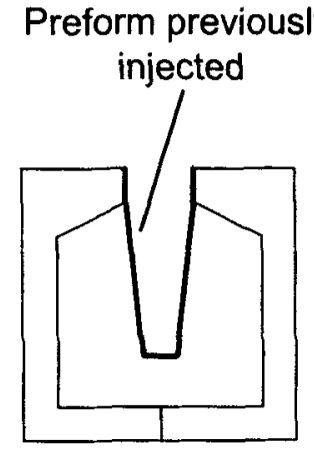

(1)
Stretching and

blowing

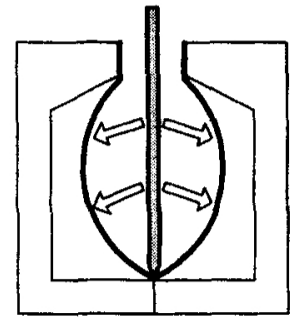

(2)

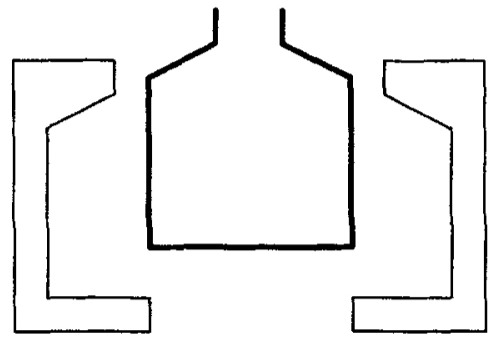

(3) (b)

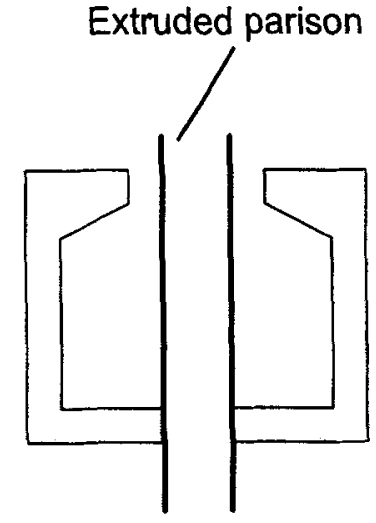

(1)

(c)

\section{Heated polymer sheet}

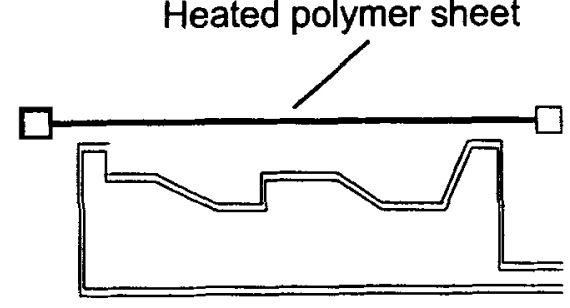

(1)

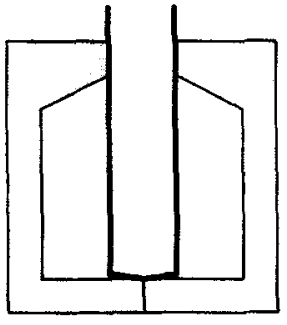

(2)

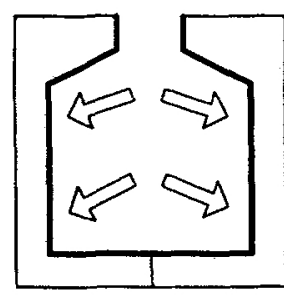

(3)

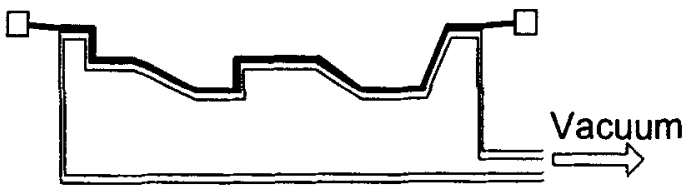

(2)

Fig. 1. Forming techniques of thin hollow thermoplastic parts: (a) extrusion blow-molding, (b) injection stretch blow-molding, (c) thermoforming.

to use the finite element method to examine the 3D membrane inflation problem. The authors considered triangular linear membrane finite elements in which strains and stresses are uniform. Then the incompressibility assumption is immediately taken into account, recalculating the parison thickness. Feng and co-workers $(6,7)$ considered the free and confined inflations of neo-Hookean and Mooney-Rivlin flat membranes. In the viscoelastic field, Wineman $(8,9)$ derived the governing equation of inflation of nonlinear viscoelastic membranes that obey a $\mathrm{K}-\mathrm{BKZ}$ constitutive equation, and Feng (10) used an integral Christensen model to study the behavior of a viscoelastic circular plane membrane. More recently, Khayat et al. (11) studied the stability of cylinders subjected to both stretching and internal pressure. Using a dynamic approach. Verron et al. extensively examined the case of spherical membrane inflation both analytically (12) and numerically (13).

The first two papers explicitly focusing on the simulation of the industrial processes were produced by Williams (14), which compared experimental and numerical results on the thermoforming of poly(methyl)methacrylate, and by Petrie and Ito (15), which demonstrated the efficiency of numerical calculations on cylindrical parisons to estimate the final thickness of 
molded parts. We can also cite the work of Ryan and Dutta $(16,17)$ : they examined the dynamic of highdensity polyethylene blow molding, highlighting the contact evolution and the influence of blowing pressure on the final geometry. These works are limited to axisymmetric problems so that the governing differential equations are directly solved by appropriate numerical methods. The three-dimensional simulation of forming processes necessitates more complex procedures, such as the finite element method (FEM). In 1989, Zamani et al. (18) reviewed publications dealing with the application of the FEM in the thermoforming process simulation. In this paper, we mention only the more recent works on the subject. One of the major contributions is by deLorenzi and Nied. They simulated both blow-molding and thermoforming processes for two-dimensional (19) and three-dimensional (20) shapes. Using experimental observations, they assumed that the parison is membrane-like and used hyperelastic constitutive equations (Mooney and Ogden models) for the behavior of thermoplastics. They solved both isothermal and non-isothermal equations using linear triangular and quadrilateral finite elements. At the same time, Charrier and co-workers used the same approach with both hyperelastic (21) and viscoelastic (22) material behaviors. More recently, Khayat and Derdouri used a hybrid finite element/finite difference method to solve the governing two-point boundary value problem of confined inflation of rubberlike membranes (23). Their results are successfully extended to the simulation of stretch blow molding (24). All these papers deal with a quasi-static treatment of the problem. Some authors prefer to consider dynamic equations and solve these equations with explicit time integration procedure classically used for metal stamping simulation. This is the case of Bourgin et al. (25) and Marckmann et al. (26).

In this paper, we report on a finite element procedure for the three-dimensional simulation of blowmolding and thermoforming processes. The dynamic part of the problem is taken into account and we use an explicit time integration scheme to solve temporal equations. The parison is assumed to be an incompressible hyperelastic rubberlike membrane. The sticking contact assumption between the parison and the mold is made. In order to improve our procedure, an efficient contact algorithm is coupled with an adaptive mesh refinement procedure.

In Section 2, the governing equations, constitutive equations, and boundary conditions are given. The finite element procedure is presented in Section $\mathbf{3}$. In that section, spatial and temporal discretization methods are briefly described, and the more special aspects such as contact treatment and adaptive mesh refinement are detailed. Three numerical examples are described in Section 4. Two of these examples study the thermoforming process and the last focuses on extrusion blow molding. They demonstrate the capability of our procedure to simulate industrial processes. Finally, concluding remarks are given in Section $\mathbf{5}$.

\section{PROBLEM FORMULATION}

In this part, governing equations of the confined inflation of rubberlike membranes are briefly recalled. For more details on theoretical aspects, the reader can refer to (13).

As mentioned in the bibliographic review, the heatsoftened parison is usually assumed to be a membrane. Its position can be therefore described in relation to the position of its mid-surface, and its thickness becomes a function of the mid-surface position. The membrane is submitted to a uniform pressure difference denoted $P(t)$. The Principle of Virtual Work is written in a hybrid Lagrangian-Eulerian form:

$$
\begin{array}{rr}
\int_{V_{0}} \partial \mathbf{u}(t) \rho_{0} \ddot{\mathbf{u}}(t) d V_{0}=-\int_{V_{0}} \partial \mathbf{E}(t): \mathbf{s}(t) d V_{0}+ \\
\int_{\partial V} \partial \mathbf{u}(t) P(t) \mathbf{n}(t) d S & \forall \partial \mathbf{u}(t)
\end{array}
$$

In this equation, $V_{0}$ and $\partial V$ are respectively the volume of the undeformed membrane and the boundary surface of the deformed membrane, $\rho_{0}$ is the constant mass density and $\mathbf{n}(t)$ is an external normal vector to the deformed membrane. $\ddot{i}(t)$ stands for the acceleration vector, and $\partial \mathbf{u}(t)$ is a compatible virtual displacement vector. Tensors $\mathbf{E}(t)$ and $\mathbf{S}(t)$ are respectively the Green-Lagrange strain tensor and the second Piola-Kirchhoff stress tensor. In $E q 1$, the left-side term represents the virtual work of inertia forces, the first right-side term stands for the virtual strain energy stored by the membrane, and the second rightside term is the virtual work done by the inflating pressure acting on the deformed body.

Thermoplastics heated far above their glass transition temperature are usually considered as rubberlike materials (20). Rubberlike materials are slightly viscoelastic, highly nonlinear and incompressible (27). Therefore, authors implement hyperelastic and/or nonlinear viscoelastic constitutive equations in their numerical procedures (18). In our work we consider the two types of behavior, but only the implementation of hyperelastic constitutive equations is presented here, because the numerical examples we found in literature were all in the elasticity field (see (13) for more details on viscoelasticy implementation). In order to use a hyperelastic constitutive relation, we have to define the form of the strain energy function. Here, we adopt the classical Mooney form of $W(28)$. There are only two material parameters, $c$ and $\alpha$, and the strain energy function is given by:

$$
W=c\left[(\operatorname{trace} \mathbf{C}-3)+\frac{\alpha}{2}\left(\operatorname{trace}^{2} \mathbf{c}-\operatorname{trace} \mathbf{c}^{2}-6\right)\right]
$$

where $\mathbf{C}$ is the right Cauchy-Green deformation tensor related to the Green-Lagrange strain tensor by the following relation:

$$
\mathbf{C}=\mathbf{2} \mathbf{E}+\mathbf{I}
$$


in which I is the identity tensor. The corresponding stress-strain relation can be cast in the following form:

$$
\mathbf{S}=-p \mathbf{C}^{-1}+2 c\left[\mathbf{I}+\alpha\left(I_{1} \mathbf{I}-\mathbf{C}\right)\right]
$$

where $p$ is the hydrostatic pressure relative to the incompressibility assumption.

Now, we examine the displacement boundary conditions. First we consider the initial boundary conditions. Initially the parison is assumed to be free; only clamped parts of it are constrained and will not move during the process simulation. The second type of boundary condition is due to contact between the parison and the mold. During the process, the heated parison comes in contact with the cold mold surface. Then the polymer rapidly cools down and its properties change: the membrane becomes stiffer and the blowing pressure is not sufficient to continue to deform it. Thus, each point of the parison in contact with the mold becomes fixed and its displacements are no longer unknown. Noting $t_{c}$ the contact time of a parison point $P$ and $t_{f}$ the final blowing time, the displacement vector of $P$ at current time $t, \mathbf{v}_{\mathbf{p}}(t)$, is given by:

$$
\mathbf{U}_{\mathbf{P}}\left(t_{c} \leq t \leq t_{f}\right)=\mathbf{u}_{\mathbf{P}}\left(t_{c}\right)
$$

and consequently its position $\mathbf{x}_{\mathbf{P}}(t)$ is fixed:

$$
\mathbf{x}_{\mathbf{p}}\left(t_{c} \leq t \leq t_{f}\right)=\mathbf{x}_{\mathbf{m}}
$$

where $\mathbf{X}_{\mathbf{m}}$ is the position of the coinciding point on the mold surface. It is noteworthy that the problem becomes a smaller and smaller free inflation problem as the parison comes in contact with the mold.

\section{NUmERICAL PROCEDURE}

In this section we present the finite element procedure adopted to solve the problem described above. Since the free inflation simulation aspects are detailed elsewhere (13), we briefly cover them and we highlight the more special aspects related to the industrial processes simulation.

\subsection{Finite Elements and Time Integration Scheme}

The parison is discretized in linear triangular finite elements and the Principle of Virtual Work Eq 1 is written for each element. The elementary contributions are assembled on the parison mesh and the system to be solved can be written as:

$$
\mathbf{M} \ddot{\mathbf{u}}(t)=\mathbf{F}^{\text {ext }}(t)-\mathbf{F}^{\text {int }}(t)
$$

in which $\mathbf{M}$ is the mass matrix constant in time, $\ddot{\mathbf{U}}(t)$ is the nodal acceleration vector $(\mathbf{U}(t)$ stands for the vector of nodal displacements), and $\mathbf{F}^{\text {rext }}(t)$ and $\mathbf{F}^{\text {int }}(t)$ are respectively the external and internal nodal force vectors.

Equation 7 is a system of ordinary nonlinear differential equations of the unknown $t$. In order to solve it, the time is discretized using the explicit second-order central difference method. This scheme is conditionally stable so that a small time step has to be adopted. Moreover, the resolution algorithm is greatly improved by the use of the special lumping technique proposed by Zienkiewiecz and Taylor: the mass matrix $\boldsymbol{M}$ can be approached by a diagonal mass matrix denoted $\mathbf{m}^{\prime}$ (29). Thus the system to be solved becomes diagonal and each term of the nodal displacement vector, $U_{i}$ (degree of freedom $i$ ), at time $t+\Delta t$ depends only on its previous values at times $t$ and $t-\Delta t$ :

$$
\begin{aligned}
U_{i}(t+\Delta t)=\frac{\Delta t^{2}}{M_{i t}^{\prime}}\left[F_{i}^{\text {ext }}(t)\right. & \left.-F_{i}^{\text {int }}(t)\right] \\
+ & 2 U_{i}(t)-U_{i}(t-\Delta t)
\end{aligned}
$$

In order to initiate the scheme, we impose that displacements and velocities of all nodes are equal to zero initially.

\subsection{Contact Treatment}

As mentioned above, the parison is supposed to stick to the mold. Hence the contact problem is reduced to two steps: the detection of the contacted parison nodes and their projection on the mold for the rest of the simulation.

First, in the detection step, we list the nodes that came through the mold during the last time increment. Consider a parison node $i$ which occupies the position $N$ at the discrete time $t_{n^{*}}$ Note $N^{\prime}$ its position at the previous discrete time $t_{n-1}$. In order to perform the detection, the mold is divided in 3-nodes triangular elements. We note (DEF) one of these mold elements. Figure 2 presents the problem geometry. The goal of the detection step is to determine if the trajectory of the node $i$ crossed the element $(D E F)$ during the time step $\Delta t=t_{n}-t_{n-1}$. Geometrically it is equivalent to determine if the intersection point, noted $M$,

Fig. 2. Geometry of the contact problem.

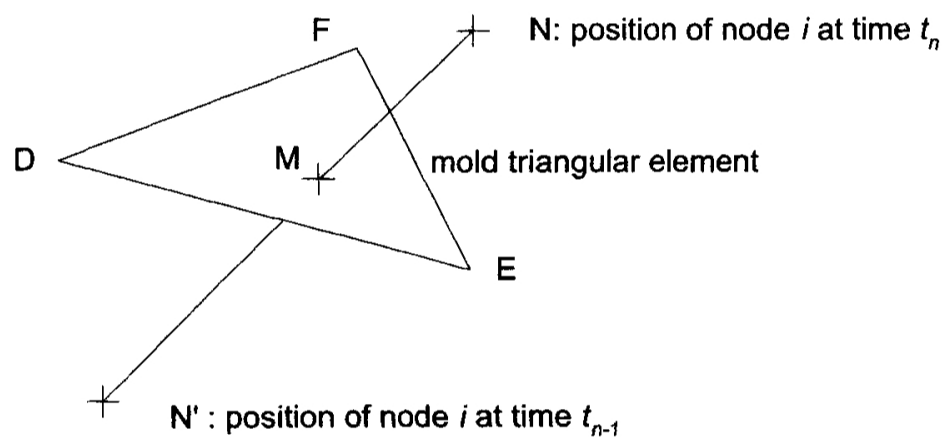


between the straight line $(L)$ that passes through $N$ and $N^{\prime}$ and the plane $(P)$ that passes through $D, E$ and $F$ is both between $N$ and $N^{\prime}$, and in the triangle $(D E F) . M$ lies on the line $(L)$ if:

$$
\mathbf{N}^{\prime} \mathbf{M}=\boldsymbol{\beta} \mathbf{N}^{\prime} \mathbf{N}
$$

where $\beta$ is a real number, and on the plane $(P)$ if:

$$
\mathbf{D M}=\gamma \mathbf{D E}+\delta \mathbf{D F}
$$

in which $\gamma$ and $\delta$ are two real numbers. Using these two equations, the problem is reduced to a linear system of three equations written under the following vectorial form:

$$
\beta \mathbf{N} \mathbf{N}^{\prime}+\gamma \mathbf{D E}+\delta \mathbf{D F}=\mathbf{D N}
$$

where $\beta, \gamma$ and $\delta$ are the three unknown coefficients. Finally, the node $i$ came in contact with element $(D E F)$ during the time step if the previous coefficients verify simultaneously the four following conditions:

$$
\begin{aligned}
0 \leq \beta \leq 1, \quad 0 \leq \gamma \leq 1, \quad 0 & \leq \delta \leq 1, \\
& 0 \leq 1-\gamma-\delta \leq 1
\end{aligned}
$$

Second, after having tested all nodes with all mold elements, the contacted nodes list is established, and we must project these nodes onto the mold. To simplify the discussion, we consider the previous node $i$ and we suppose that it came in contact with the mold on (DEF). Moreover we assume that the motion of $i$ between the two geometric points $N^{\prime}$ and $N$ is linear during the time step $\Delta t$. This assumption is not very restrictive because the time step is very small owing to the explicit temporal integration scheme adopted. Consequently, the projection point is $M$ (defined above) and the projection of the node is made using the coefficient $\beta$ which places $M$ between $N^{\prime}$ and $N$. The node $i$ is then fixed at this new position until the end of the simulation.

Recall that in contact problems, the number of operations and tests is very large; note that this method is very powerful because there is no assumption on the mold elements' size.
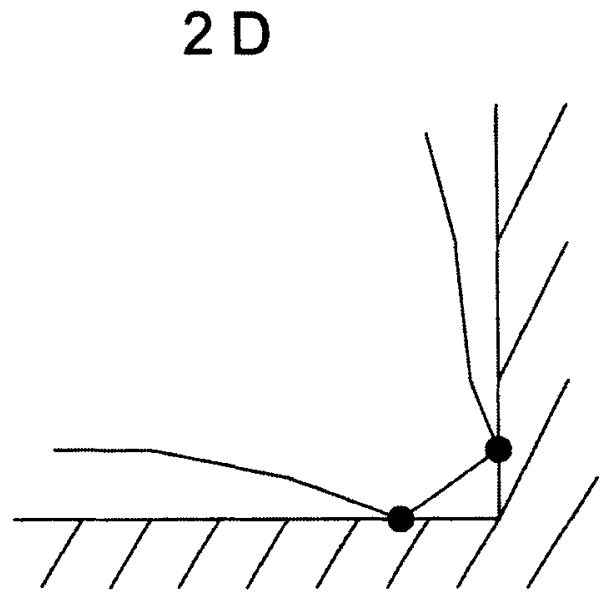

\subsection{Adaptive Mesh Refinement Scheme}

One of the major problems with the use of triangular linear finite elements is that these elements always stay plane. Taking into account their large deformations, it becomes impossible to closely simulate geometrical changes of the parison near mold edges or corners without using many finite elements. Such difficulties are illustrated in Fig. 3. In order to overcome this difficulty, some authors present the mesh refinement as an interesting perspective, without implementing it (20).

In this section, we present the refinement criterion and the refinement algorithm that we implemented. As this work is one of the first examples of mesh refinement applied to polymer blowing simulation, a very simple criterion and algorithm were retained.

Because of large displacements, large strains and contact, the forming process simulation is geometrically highly nonlinear. That is the reason why we adopt a geometrical criterion based on angular difference between neighboring elements. When we decide to refine the mesh, for example each $k$ time step, each parison finite element is tested: elements that share one side with it are listed and a mean normal vector is calculated by summing every normal vector. After that, angles between each element normal vector and the mean normal vector are computed and compared with a user-defined limit value. If one (or more) of these angles is greater than the limit value, the corresponding element is divided before the next time step. Moreover, the user can define an elementary limit surface to prevent the creation of too small elements. Figure 4 shows a tested element: this central element is numbered 1 and the neighboring elements are numbered from 2 to 4 .

As mentioned above, the goal of this part of the work is to evaluate the capability of adaptive mesh refinement in polymer forming simulation context. Thus we decided to adapt and implement the algorithm developed by Nambiar et al (30) because it seems to be both simple to implement and efficient. Their technique is
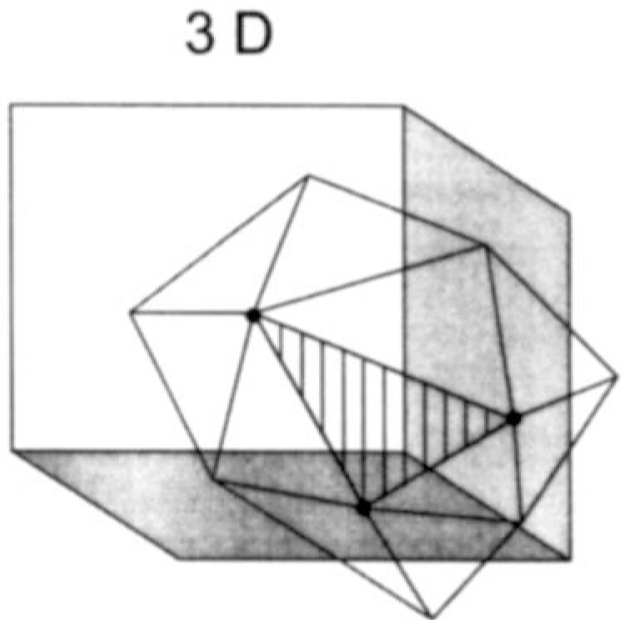

Fig. 3. Contact problem in mold corners with a coarse mesh. 


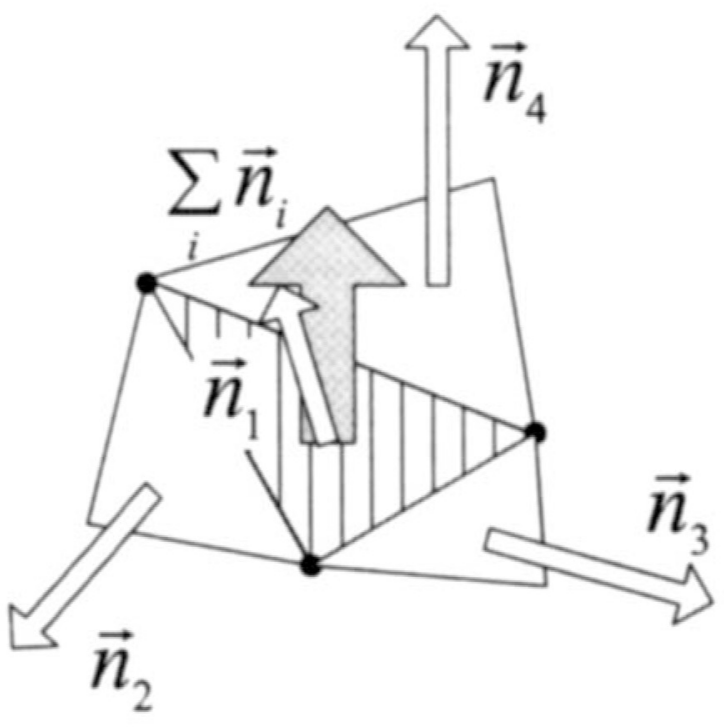

Fig. 4. A group of finite elements tested for refinement.

based on the addition of a node only at the mid-side of the longest edge of refined elements. We briefly recalled the subdivision method used in Fig. 5. The method depends on three geometrical cases.

- The element to be divided has its longest edge on the boundary of the domain (see Fig. 5a). Then a new node is created at the mid-side of this edge and two new elements replace the previous one.

- The longest edge of the element to be divided is also the longest edge of the element facing it (see Fig. 5b). In this case the common side is bisected and four new triangles are created.

- The longest edge of the element to be divided is not the longest edge of the element facing it (see Fig. 5c). This case arises when one element is to be divided (triangle 1 in the figure) and its facing element is not to be divided (triangle 2 in the figure). Then the second triangle is added to the list of elements for subdivision and the procedure is applied to this element (previous case).

In order to implement this method, the triangles to be subdivided are sorted in order of increasing length of their longest edge. The subdivision algorithm is presented in Fig. 6. More details on this method are given in the original article of Nambiar et al.

\section{NUMURICAL EXAMPLES}

In order to validate the free inflation part of the present formulation, we extensively examined elsewhere the deformation of spherical and ellipsoidal membranes (13). In this paper we focus on the capability of our program to simulate industrial processes including the use of contact and adaptive remeshing (if necessary) procedures.

\subsection{Cylindrical Shape Thermoforming}

This problem was previously studied by deLorenzi and Nied both experimentally and numerically (20).

We consider a deep conical cylinder obtained by vacuum forming. The material is high-impact polystyrene and the undeformed polymer sheet is circular. The sheet radius and thickness are respectively $129.54 \mathrm{~mm}$ and $0.254 \mathrm{~mm}$. The mold geometry and mesh are shown in Fig. 7. Because of the problem symmetry, only a quarter of the structure is studied. The mold is discretized in 63 triangles (see Fig. 7b). Following the experimental work of Schmidt and Carley (31), high-impact polystyrene behavior can be modeled by a modified Mooney-Rivlin strain energy function given by:

$$
W=A_{01}\left(I_{1}-3\right)+A_{02}\left(I_{2}-3\right)^{2}
$$

in which:

$$
A_{01}=0.143 \mathrm{MPa}, A_{02}=2.2 \times 10^{-6} \mathrm{MPa}
$$

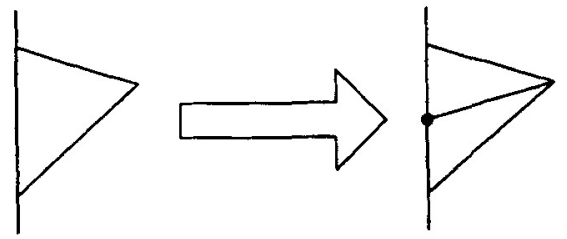

(a)
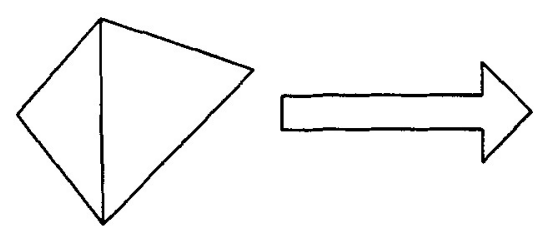

(b)
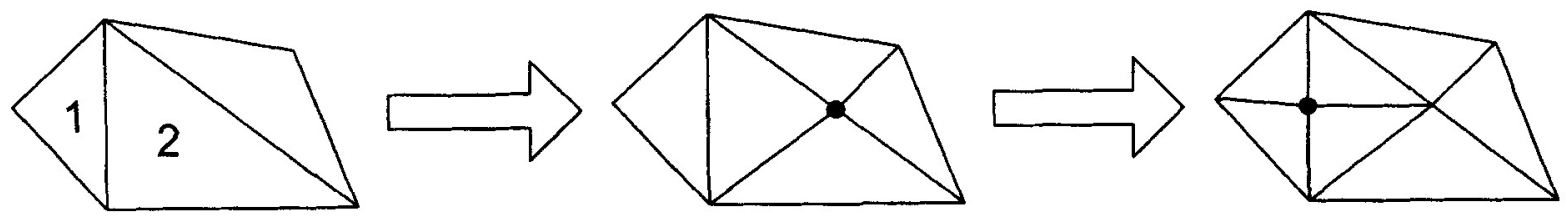

(c)

Fig. 5. The three methods of element subdivision. 


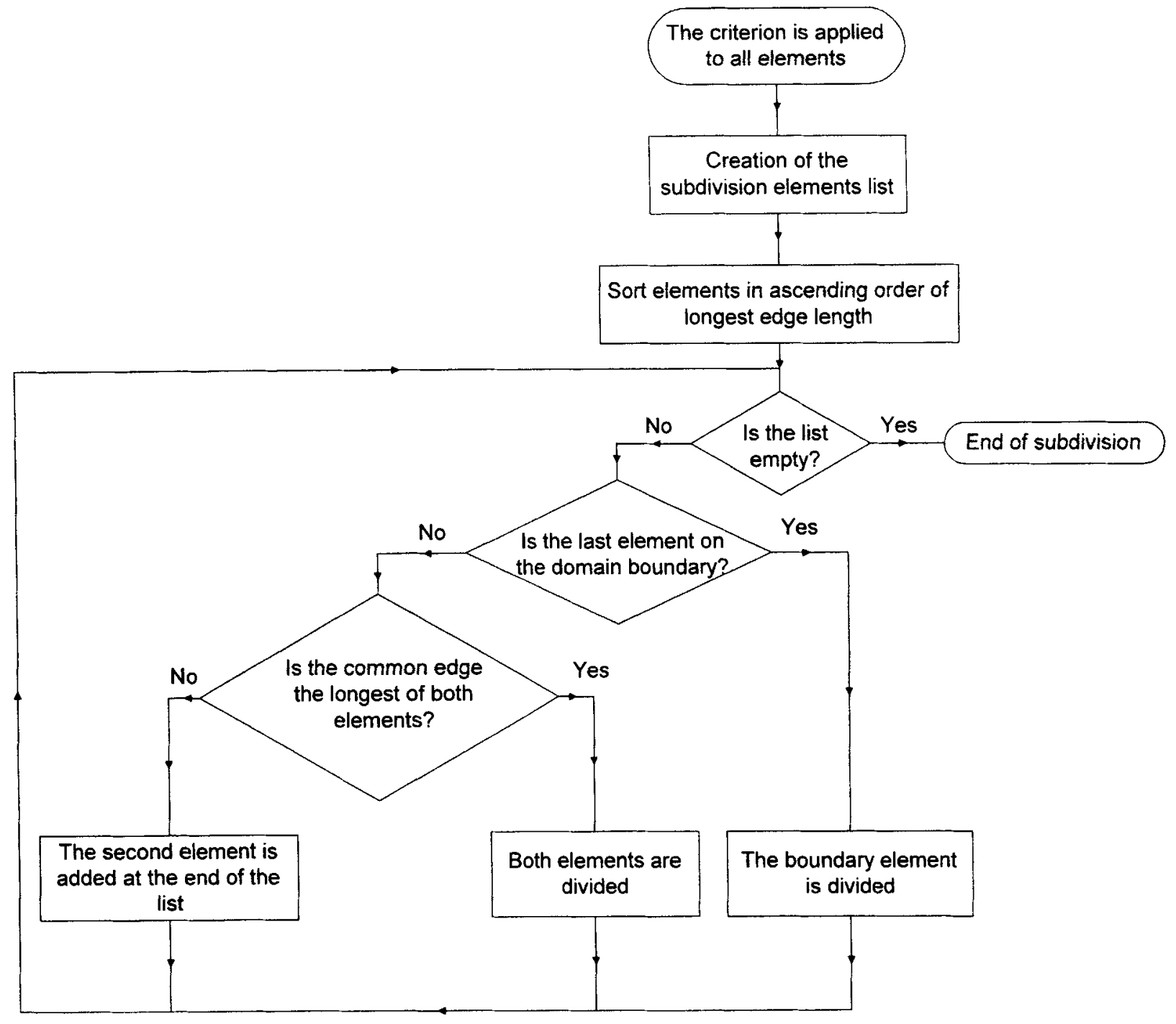

Fig. 6. Adaptive mesh refinement algorithm.

The number of finite elements on the initial mesh is 27. This number is small and the mesh is relatively coarse. Therefore, we use the mesh refinement procedure presented above: at the end of the simulation, there are 996 finite elements. Figure 8 presents four stages of the dish thermoforming. Note the quality of the final mesh (Fig. 8d): only the use of a mesh refinement procedure can produce such an appropriate element distribution. DeLorenzi and Nied experimentally obtained wall thickness in the formed shape and principal extension ratios by measuring the deformation of circles printed on the initial parison. Thus we compare our numerical results with their measures in the following figures:

- Figure 9 shows the non-dimensional thickness, $h / h_{0}\left(h\right.$ and $h_{0}$ are respectively the final and initial thickness), versus the curvilinear distance from the axis of symmetry,
- Figure 10 presents the principal stretch ratios (radial $\lambda_{r}$ in Fig. 10a and circumferential $\lambda_{\theta \theta}$ in Fig. $(O b)$ versus the distance from the center of the sheet in the undeformed state.

Thickness calculations agree well with experimental results especially at the bottom of the cylinder. At the outside edge of the dish, for curvilinear distances greater than $250 \mathrm{~mm}$, numerical thickness values are underestimated. The major reason for this difference may be due to the final coarse mesh on the edge. In fact the corresponding finite elements entered in contact very soon and were not subdivided enough to give sufficiently accurate results. In Fig. 10 the calculated stretch ratios seem to be less in agreement with experimental results. Figure $10 a$ shows that radial stretch ratio is overestimated on dish edge and underestimated near the symmetry axis (25\%). For the circumferential stretch ratio, the results are good at the edge of the 


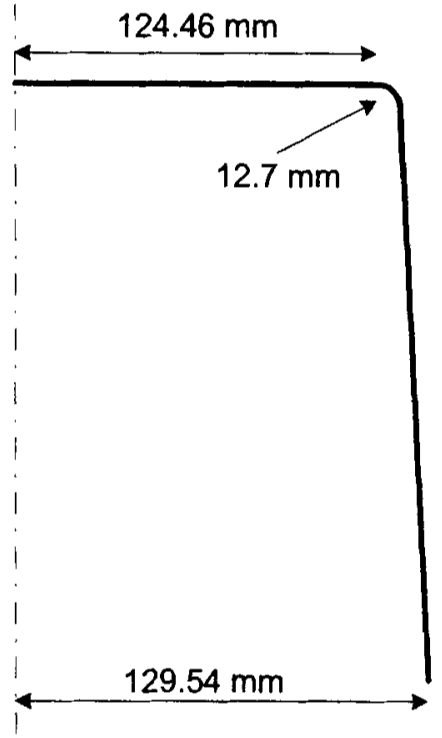

(a)

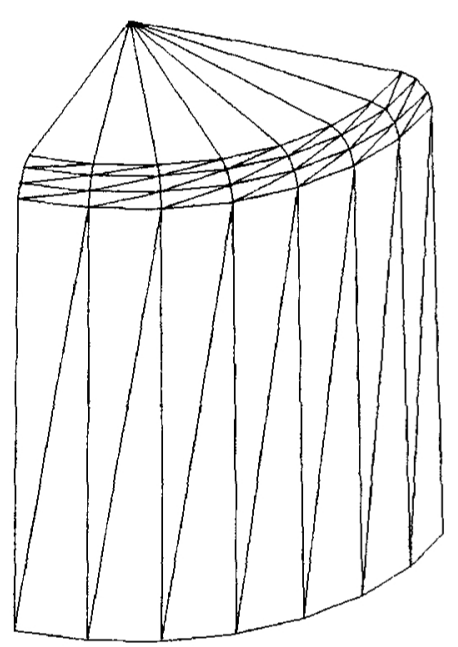

(b)

Fig. 7. (a) Mold geometry, (b) mold mesh.

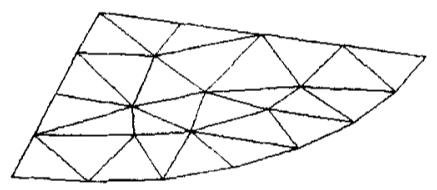

(a) 27 finite elements

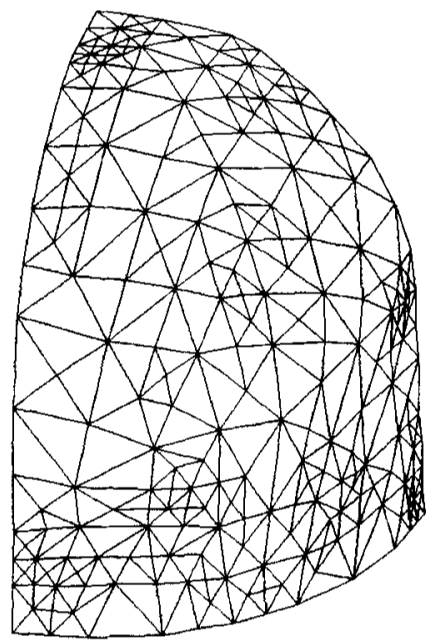

(c) 429 finite elements

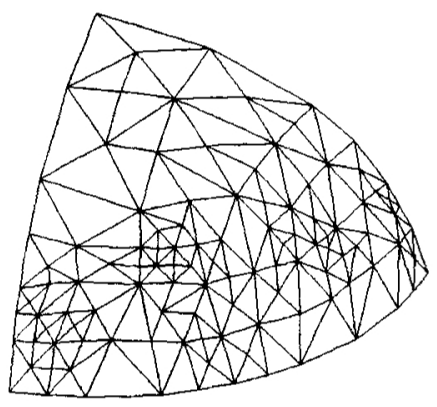

(b) 201 finite elements

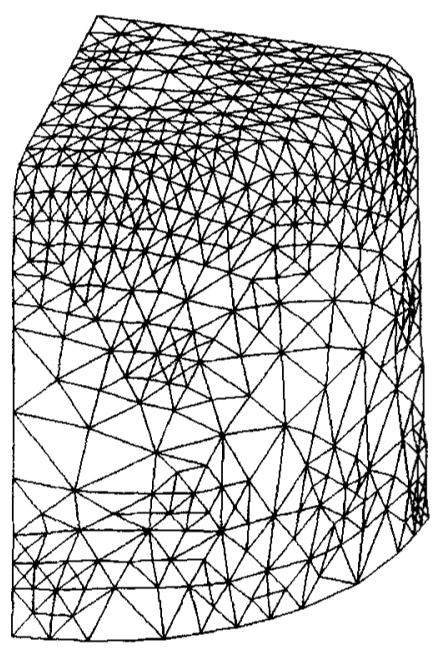

(d) 996 finite elements

Fig. 8. Four stages of vacuum forming simulation of a cylindrical dish. 
Fig 9. Non-dimensional thickness in thermoformed cylinder: (I) experimental results from (20), (O) our numerical results.

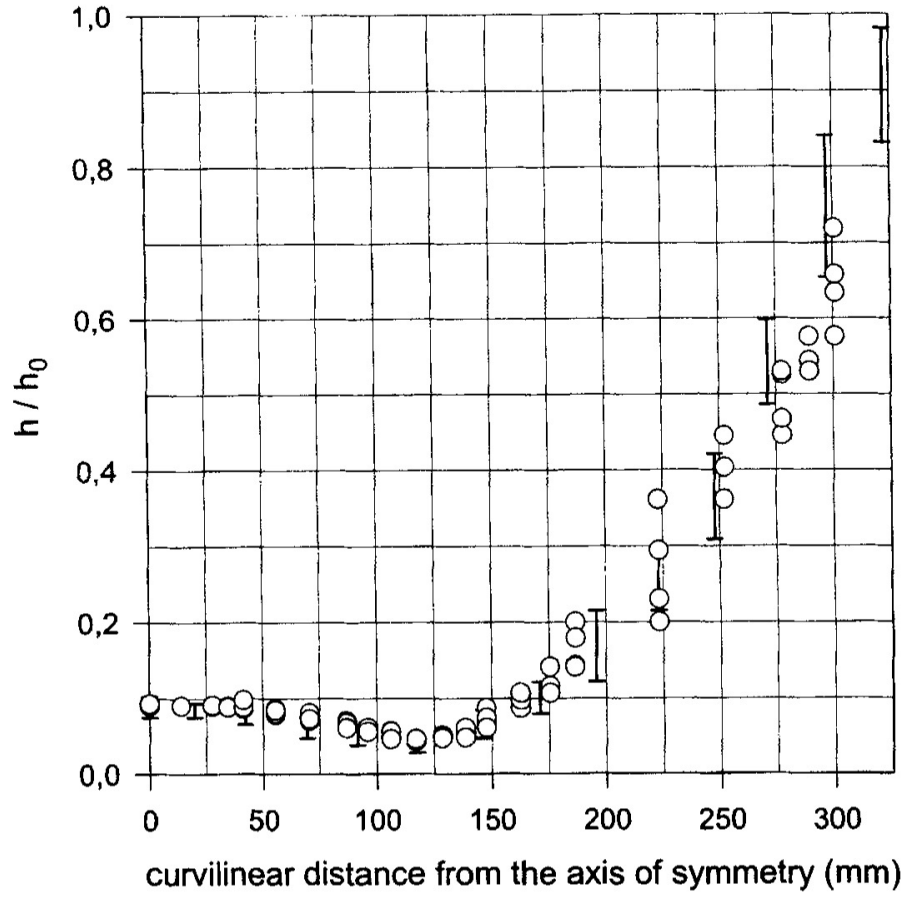

\subsection{Thermoforming of a Complex Shape}

We now examine a more academic case to intensively validate our contact and remeshing procedures.

The mold is a box with a central insert at the top. Figure 11 presents the mold geometry (Fig. 11a) and the mold mesh of 26 triangular elements (Fig. 11b). The mold is a 3D non-convex shape. Non-convexity often involves problems during contact treatment: an element can be in contact with the mold even if its three nodes did not come through the mold wall during the

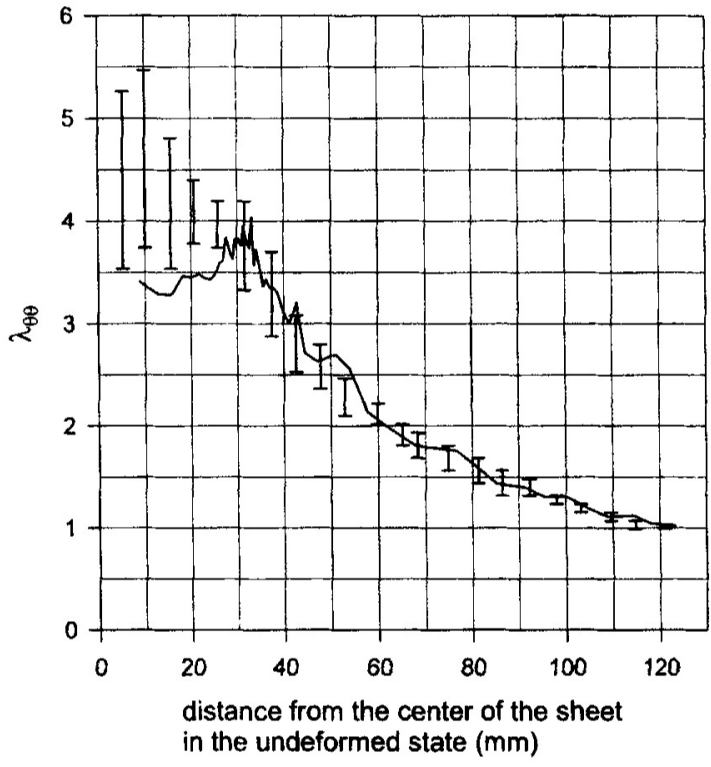

(b)

Fig. 10. (a) Radial stretch ratio in thermoformed cylinder, (b) circumferential stretch ratio in thermoformed cylinder: (I) experimental results from (20), ( $\rightarrow$ ) our numerical results. 


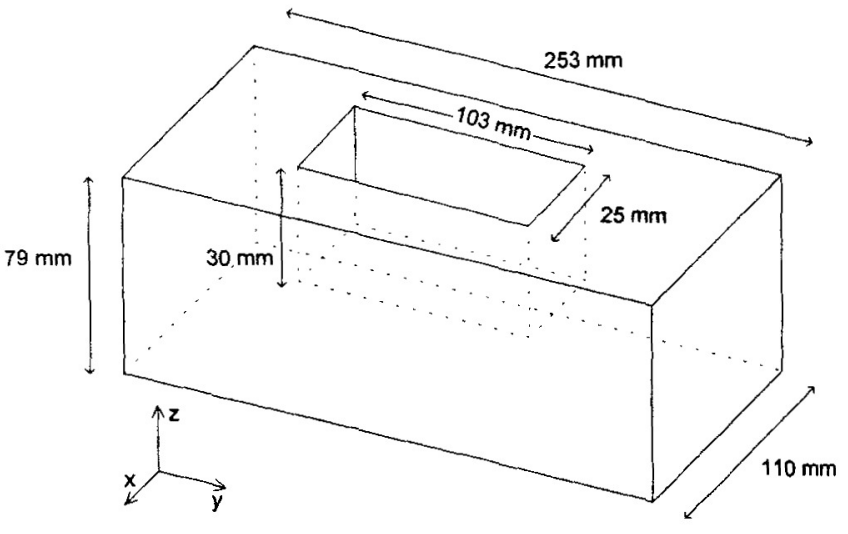

(a)

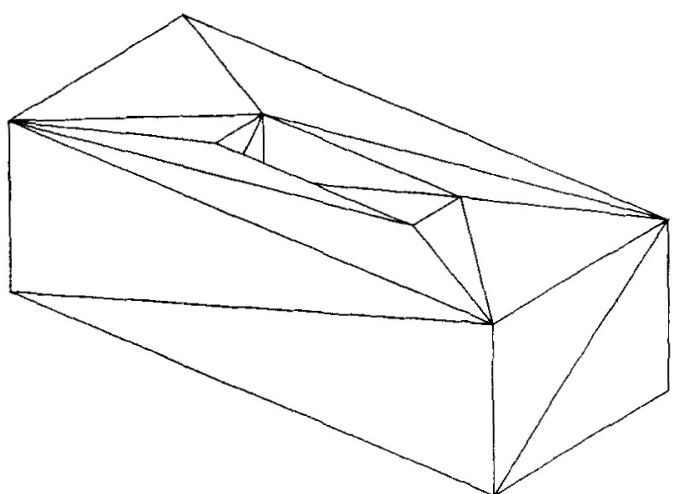

(b)

Fig. 11. (a) Mold geometry for the box with insert, (b) mold mesh.

last time step. Then, using automatic remeshing, it becomes possible to create a new node that is already outside the mold. Consequently, the present example is a severe test for our procedures. The initial parison is a rectangular sheet whose thickness is set to $2 \mathrm{~mm}$. Its dimensions are the same as those of the box bottom and its four edges are clamped. The corresponding boundary nodes are fixed during the simulation.

Four stages of the process are displayed in Fig. 12. The initial mesh has 128 finite elements (see Fig. 12a) and the final mesh contains 3752 finite elements (see see Fig. 12d). The precision of the final mesh in the vicinity of corners and edges of the insert compared with the initial coarse mesh is to be noted. Figure 13 shows wall thickness in the molded part and Fig. 14a and $b$ present the wall thickness and the final shape in the symmetry planes $(x z)$ and ( $y z)$, respectively. These symmetry planes are identified in Fig. 11 a.

The present example highlights the capability of our procedures: the contact algorithm can handle with non-convex molds, and the remeshing procedure allows the use of initial coarse meshes in order to reduce the user's effort (it is well known that one of the major obstacles in using a finite element analysis
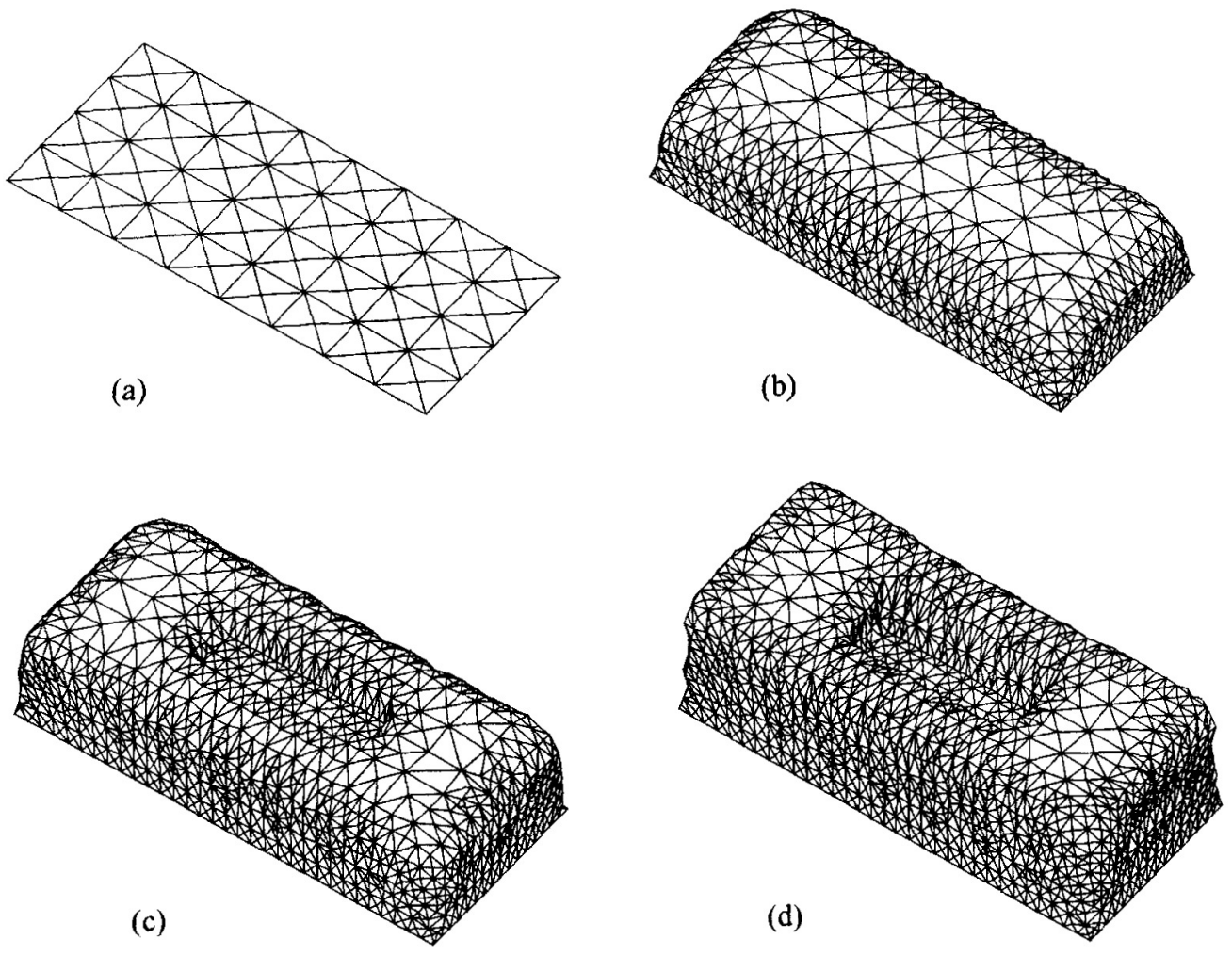

Fig. 12. Four stages of thermoforming of a box with insert. 


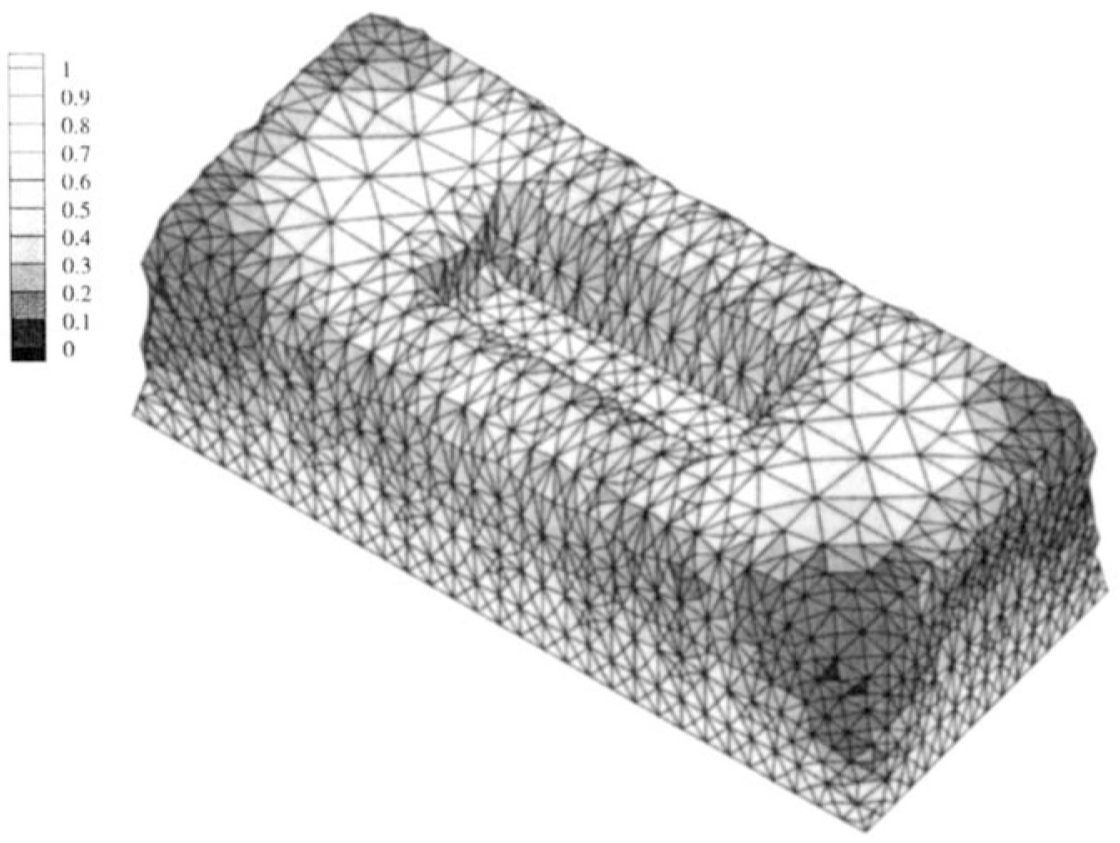

Fig. 13. Thickness distribution in the thermoformed box with insert.

package is the design of an initial locally refined mesh, which is necessary to obtain a confident solution).

\subsection{Blow Molding of a Bottle With Fandle}

The last example focuses on the extrusion blowmolding process. We examine the case of a soap bottle with handle. This problem was previously studied by Khayat and Derdouri (24) both experimentally and numerically.

During the industrial process, the bottle parison is extruded before being pinched by the two parts of the

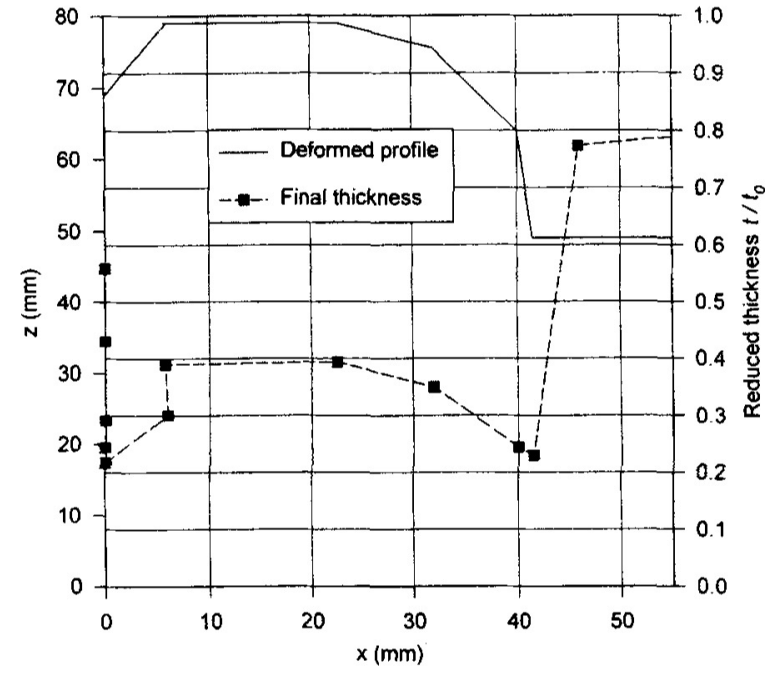

(a) mold. Then, the bottom and edges of the bottie and the edges of the handle are clamped. The geometry and mesh of the corresponding semi-parison after the extrusion stage are presented in Fig. 15. This shape was obtained by Khayat and Derdouri just before inflation began. in practice, all flattened parts are cut out after the blowing stage. The bottle height and maximum radius are, respectively, $230 \mathrm{~mm}$ and $40 \mathrm{~mm}$. Initial thickness is assumed to be uniform and equal to $2.25 \mathrm{~mm}$. The initial parison is meshed with $802 \mathrm{fi}-$ nite elements, and we do not use the mesh refinement

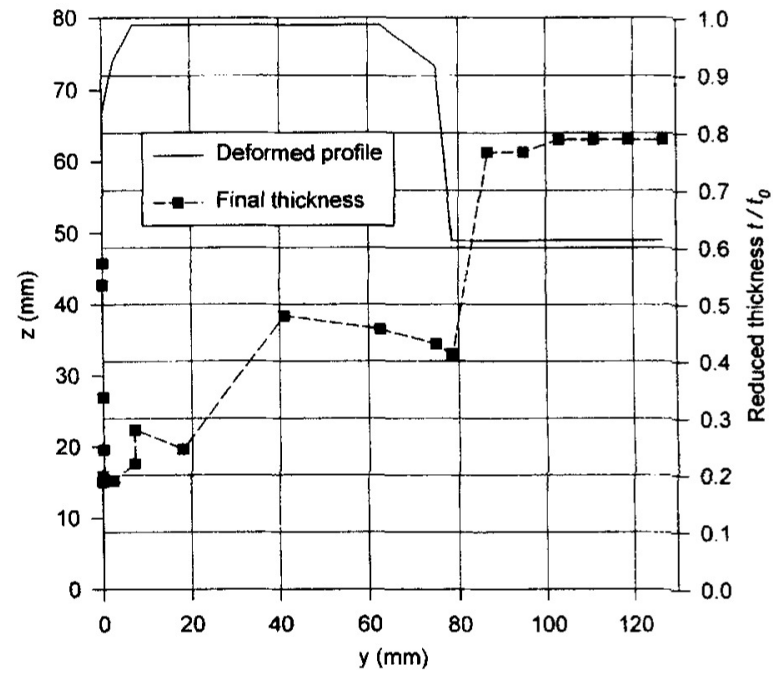

(b)

Fig. 14. Deformed profile and final thickness distribution in the symmetry planes: (a) (xz), (b) (yz). 


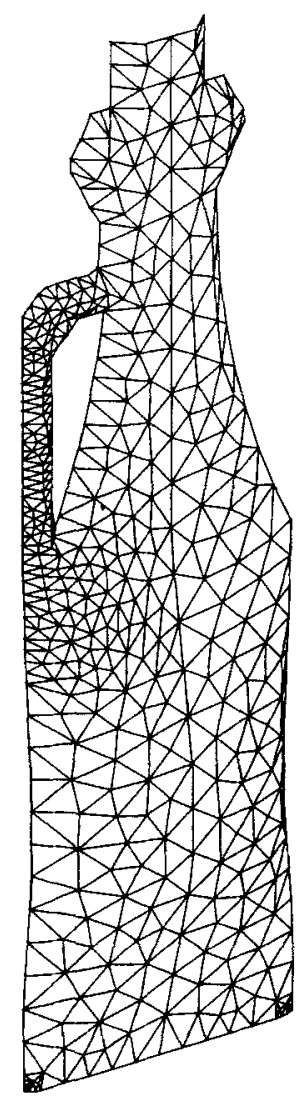

(a)

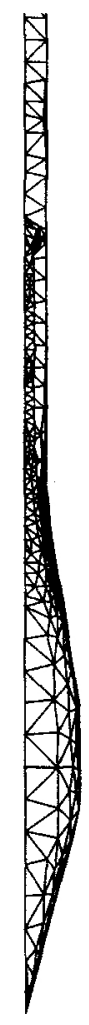

(b)
Fig. 15. Initial parison for the soap bottle: (a) at $45^{\circ}$, (b) at $90^{\circ}$.

procedure. The mold discretization is shown in Fig. 16. This mesh has 1215 triangular elements. The material is PET and it is modeled by a Mooney-Rivlin constitutive equation with (24):

$$
\alpha=0.5
$$

Figure 17 shows the final reduced thickness distribution along the bottle surface from two different views. As Khayat and Derdouri, we observe $60 \%$ thinning in the body of the bottle, $20 \%$ thinning in the neck and approximately $50 \%$ in the bottom (see Fig. 17a). In the handle, the thinning is mostly uniform in the order of $50 \%-60 \%$. Comparisons between our results and both numerical and experimental results of Khayat and Derdouri are reported in Fig. 18. Figures 18 $a$ and $b$ show final thickness distribution along lines A-B-C and $a-b-c$, respectively. These lines are identified in Fig. 17. The curves display the final thickness distribution versus the curvilinear distance from the bottom to the top of the soap bottle. First, our results agree well with numerical results from (24) even if numerical procedures are highly different. Second, examining the comparison with experimental results for the botthe body (see Figs. 17a and 18a), the difference between our numerical and their experimental results changes from $10 \%$ to $20 \%$ following the line A-B-C from the bottom to the neck. Along the line a-b-c (see

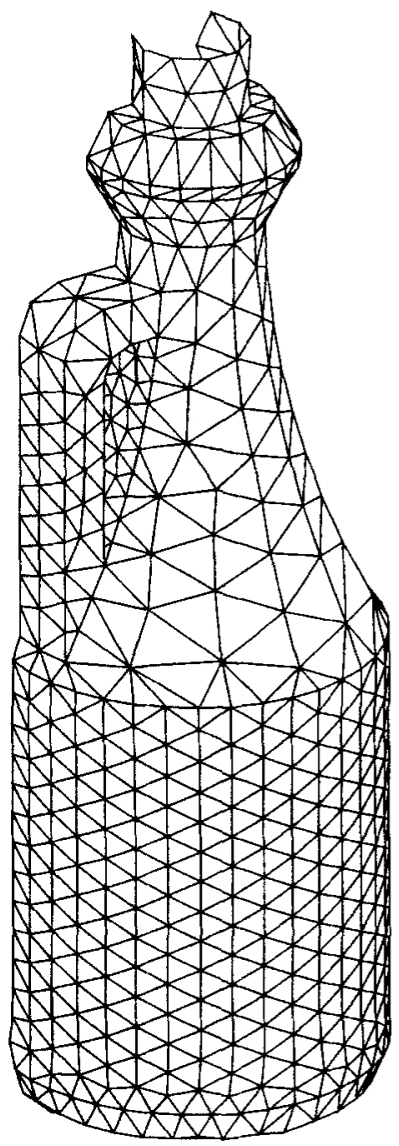

Fig. 16. Mold mesh for the bottle blow-molding.

Figs. $17 b$ and $18 b$ ) the differences are very slight at the bottom (point a), 50\% in the body (point b) and they oscillate around the experimental curve in the handle (point c). These oscillations are due to the mesh behavior during the contact: in the handle some elements enter into contact very early without thinning and some others enter into contact very late. As mentioned by Khayat and Derdouri, the differences might be due to the assumed uniform initial thickness distribution. It is well known that the final thickness distribution depends highly on the initial thickness distribution (15). In fact, as the parison is extruded and clamped, we can affirm that the hypothesis of uniform initial thickness is false and that it is the main cause of the difference between experimental and numerical results.

\section{Concluston}

A dynamic nonlinear finite element procedure has been developed to simulate the blow-molding and thermoforming industrial processes. The heated polymer parison is assumed to be an incompressible hyperelastic rubberlike membrane and is meshed with linear triangular elements. The use of an explicit time integration scheme reduces the problem to a vectorial iterative equation and highly simplifies the contact and remeshing procedures implementation. 

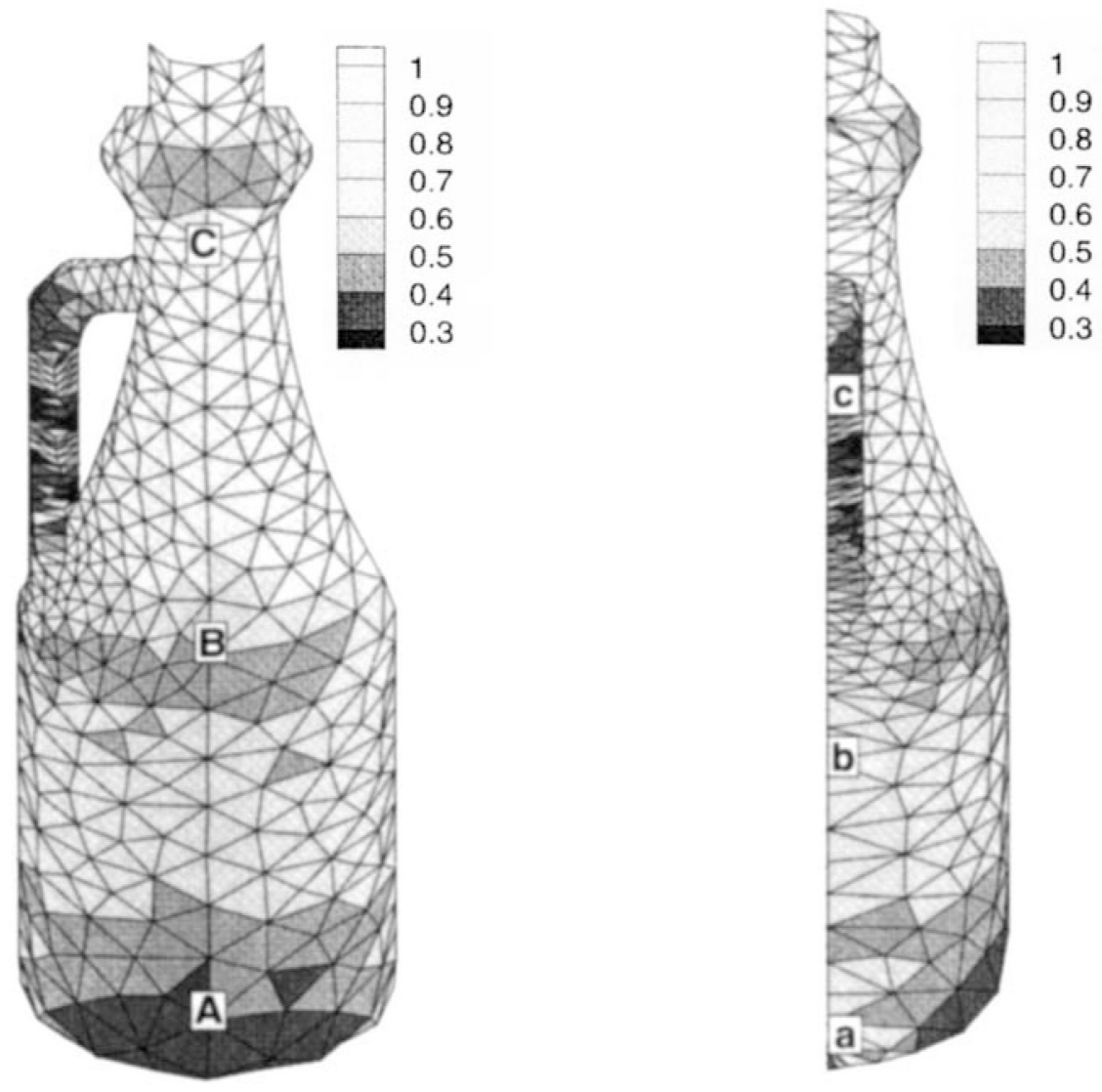

Fig. 17. Final reduced thickness distribution on the bottle.

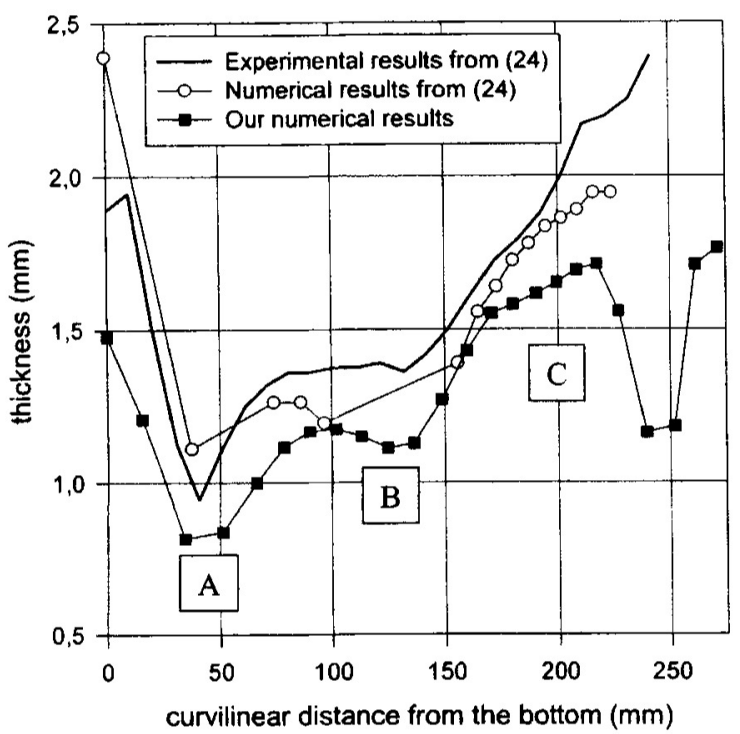

(a)

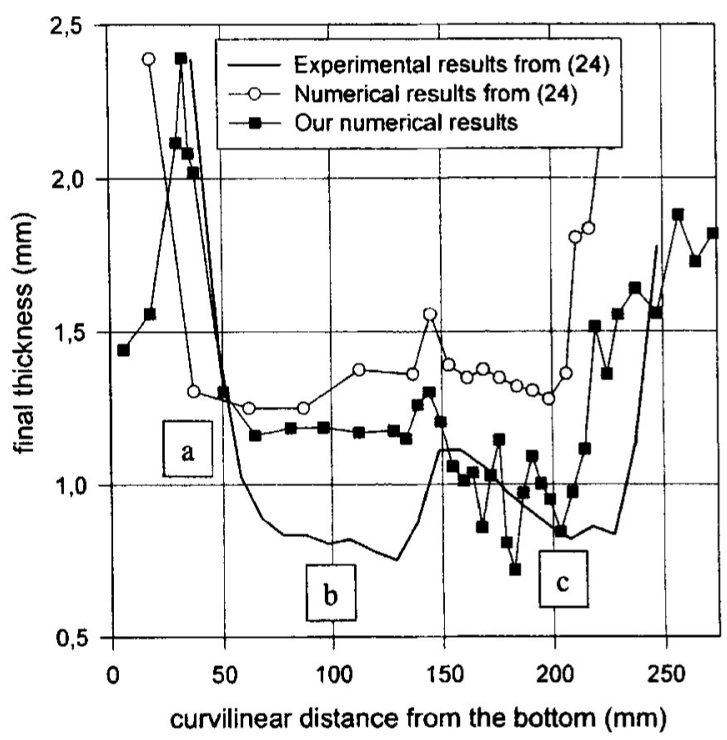

(b)

Fig. 18. (a) Final thickness distribution along A-B-C, (b) final thickness distribution along a-b-c. 
The comparison between our numerical results and different experimental and numerical results from literature is fairly good for both blow-molding and thermoforming simulations.

Further developments will be necessary to improve the quality of the results. One of the main developments will be a better knowledge of material behavior such as the influence of temperature on material stiffness. This experimental aspect will pave the way to further numerical issues.

\section{ACKNOWLEDGMENT}

The authors wish to thank Dr. A. Derdouri and Prof. R. E. Khayat for putting at their disposal numerical and experimental data relative to the soap bottle.

\section{REFERENCES}

1. D. V. Rosato and D. V. Rosato, Blow Molding Handbook, Hanser Publishers, Munich (1989).

2. J. L. Throne, Thermoforming, Hanser Publishers, Munich (1987).

3. A. E. Green and J. E. Adkins, Large Elastic Deformations, The Clarendon Press, Oxford, England (1960).

4. L. J. Hart-Smith and J. D. C. Crisp, Int. J. Eng. Sci, 5, I (1967).

5. J. T. Oden and T. Sato, Int. J. Solids Structures, 3, 471 (1967).

6. W. W. Feng and W. H. Yang, J. Appl. Mech. ASME, 40 , 209 (1973).

7. W. W. Feng and P. Huang, J. Appl. Mech. ASME, 41, 767 (1974)

8. A. Wineman, J. Non-Newtonian Fluid Mech., 4, 249 (1978).

9. A. Wineman, J. Non-Newtonian Fluid Mech., 6, 111 (1979).

10. W. W. Feng, J. Appl. Mech. ASME, 59, S29 (1992).

11. R. E. Khayat, A. Derdouri, and A. Garcia-Réjon, Int. J. Solids Structures, 28, 69 (1992).
12. E. Verron, R. E. Khayat, A. Derdouri, and B. Peseux, J. Rheol, 43, 1083 (1999).

13. E. Verron, G. Marckmann, and B. Peseux, Int. J. Num. Meth. Eng., to be published.

14. J. G. Williams, J. Strain Analysis, 5, 49 (1970).

15. C. J. S. Petrie and K. Ito, Plastics Rubber Proc., 68 (1980).

16. M. E. Ryan and A. Dutta, Polym. Eng. Sci, 22, 1075 (1982).

17. A. Dutta and M. E. Ryan, Polym. Eng. Sci., 24, 1232 (1984).

18. N. G. Zamani, D. F. Watt, and M. Esteghamatian, Int. J. Num. Meth. Eng., 28, 2681 (1989).

19. H. G. deLorenzi and H. F. Nied, Comput. Struct., 26, 197 (1987).

20. H. G. deLorenzi and H. F. Nied, in Progress in Polymer Processing, A. I. Isayev, ed., Hanser Verlag (1991).

21. J. M. Charrier, S. Shrivastava, and R. Wu, J. Strain Analysis, 24, 55 (1989).

22. S. Shrivastava and J. Tang, J. Strain Analysis, 28, 31 (1993).

23. R. E. Khayat and A. Derdouri, Int. J. Num. Meth. Eng., 37, 3773 (1994).

24. R. E. Khayat and A. Derdouri, Polym. Eng. Sci., 35, 1852 (1995).

25. P. Bourgin, I. Cormeau, and T. Saint-Martin, J. Mat. Processing Tech., 54, 1 (1995).

26. G. Marckmann, E. Verron, and B. Peseux, Rev. Européenne des Elements Finis (in french), 7, 193 (1998).

27. I. M. Ward, Mechanical Properties of Solid Polymers, Second Ed., John Wiley and Sons Ltd. (1983).

28. L. R. G. Treloar, Proc. R. Soc. Lond., A351, 301 (1976).

29. O. C. Zienkiewiecz and R. L. Taylor, The Finite Element Method. Volume I, Fourth Ed., McGraw-Hill Company (1994)

30. R. V. Nambiar, R. S. Valera, K. L. Lawrence, R. B. Morgan, and D. Amil, Int. J. Num. Meth. Eng., 36, 499 (1993)

31. L. R. Schmidt and J. F. Carley, Polym. Eng. Sci, 15, 51 (1975). 